\title{
Emitter Localization and Visualization (ELVIS): A Backward Ray Tracing Algorithm for Locating Emitters
}

\author{
Aliye Özge Kaya \\ Larry Greenstein \\ WINLAB, Rutgers University \\ \{ozgekaya, ljg\}@winlab.rutgers.edu
}

\author{
Dmitry Chizhik \\ Reinaldo Valenzuela \\ Lucent Tech, Bell Labs \\ \{chizhik,rav\}@lucent.com
}

\author{
Nader Moayeri \\ National Institute of \\ Standards and Technology \\ \{moayeri\}@nist.gov
}

\begin{abstract}
We present ELVIS, a new approach for localizing a first responder (e.g., firefighter) inside a building. We assume that the first responder emits RF energy, which undergoes multiple reflections with the walls, ceilings and floors of the building. There are $K$ receivers, each of which receives ray(s) from the first responder. Each receiver estimates the AOA (Angle of Arrival), TOA (Time of Arrival) and power of each ray. We assume that the receivers know the blueprint of the building and the electromagnetic characteristics of the construction material used in the building. We show that, based on this information, the receivers can localize the first responder to a high degree of accuracy by applying ELVIS, which is based on backward ray tracing. We have evaluated the performance of ELVIS, using both single and multiple receivers, under a variety of channel and propagation conditions. We find that, the location prediction error depends mostly on the angular resolution of the receiver antennae. One advantage of ELVIS over other RFbased localization methods is that, if AOA is estimated with high degree of accuracy, a single receiver would be sufficient to do localization in 3D. In this case one could localize $100 \%$ of the Bell Labs, Crawford Hill building within $9 \mathrm{~cm}$. With degraded accuracy, including fading and noise effects, \pm 10 degree angular error, and \pm 10 ns temporal error, one could localize $80 \%$ of the building within $10 \mathrm{~m}$ using multiple receivers.
\end{abstract}

\section{INTRODUCTION}

Consider an emergency situation like fire within a large building. It is important to localize the emergency personnel, such as police, fire-fighters etc., for both tactical and rescue purposes. Except for urban canyons, the localization problem for outdoor scenarios can be effectively solved by attaching GPS devices to the transmitters that need to be localized [1]. However, the reception of GPS signals is unreliable in most buildings. Therefore new technologies are needed to localize the emitters inside buildings.

\section{A. Prior Work}

The various approaches in the literature for localizing first responders inside buildings can be broadly classified into three categories: TOA, AOA and signal-strength-based techniques [2]. These can be realized within the domain of existing networks and technologies such as, GSM networks, $802.11 \mathrm{~b}$ networks, Bayesian-based localization or ultra-wideband (UWB) techniques. UWB provides very good resolution in the time domain which is used for localization [2]. AOA based methods require high resolution directional antennae and need at least 2 receivers to localize objects in two dimensions (2D) [2].
Signal-strength-based techniques use the fact that the distance between two nodes can be determined by measuring the received signal strength. There are multiple methods based on this approach: RADAR [3] uses empirically and theoretically determined signal strength information at multiple base stations to triangulate the user's coordinates. Bayesian techniques are applied in [4] on the signal strength of the received $802.11 \mathrm{~b}$ packets. SPS [5] is the graph of the received power as a function of direction. The through forward ray tracing predetermined SPS could be compared with the measured SPS for localization. Similarly the location of the mobile could be determined comparing the signal strength measurements at multiple GSM channels with the results obtained in the training phase [1].

Back ray tracing is discussed in [6] for ocean acoustics. Estimated angles and relative arrival times of the multipath trajectories are back-propagated using a ray tracing algorithm to localize the source.

\section{B. Our Contributions}

We propose a new algorithm, called ELVIS for localizing emitters based on backward ray tracing. Our work uses backward ray tracing for the first time for indoor emitter location prediction. ELVIS combines TOA, AOA and signalstrength-based techniques with backward ray tracing into a single framework. One advantage of our algorithm is that three dimensional (3D) localization could be done even with one receiver.

We assume that each first responder carries a beacon emitting a signal of known characteristics. We are interested in locating such an emitter placed inside a building based on signals received by one or more receivers outside of the building. The receivers measure received power as a function of AOA as well as TOA.

To assess the performance of ELVIS, it would be ideal to determine location based on data collected from high angular and temporal resolution receivers. As a preamble to making such measurements we estimate the localization accuracy based on synthetic data obtained from WISE (Wireless System Engineering), a forward ray tracing program, developed at Bell Labs [7].

As an application, we apply ELVIS to localize an emitter placed in the Bell Labs Crawford Hill building in Holmdel, 
NJ. We emulate the test environment, i.e., an emitter inside Crawford Hill, using WISE. We specify the building blue print, emitter position and transmit power, and WISE gives the received power, AOA etc. of all rays at any point in the building. We run ELVIS on this data and localize the emitter. As a practical matter, ELVIS would obtain its data from directional antennae placed at the receiver. Measurements collected using real receivers suffer from degraded accuracy because of finite angular and temporal resolution of the receivers. In addition, as ELVIS relies on using the blueprint of the building to estimate the location of the emitter, imperfect knowledge of the environment leads to errors in the amplitude of the arriving signals, perceived as "fading". Additive receiver noise is yet another source of error. We emulate such effects by perturbing the values given by WISE with errors due to fading, noise and finite resolution before running ELVIS.

The paper is organized as follows. Section II gives an overview of WISE. Section III explains the 3D backward ray tracing in ELVIS. Section IV explains how the impairments of the measurements are simulated. Section V explains in detail ELVIS. Section VI presents our simulation results.

\section{WISE (WIRELESS SYSTEM ENGINEERING)}

Given a building plan and transmitter and receiver locations, WISE measure radio-signal performance at any point in the building. WISE takes into account the layer properties of the walls and path loss. The reflection and transmission coefficients are determined based on the angle of incidence and layer properties at each interaction with a wall. WISE simulates the ray traces at the receiver as in Figure 1. For each received ray, WISE computes the AOA, the delay and the power. The power of a particular ray is given by:

$$
P_{R}=G_{T} G_{R}\left(\frac{\lambda}{2 \pi d}\right)^{2} \prod_{m=1}^{M} t_{m} \prod_{k=1}^{K} r_{k} P_{T}
$$

where $d$ is the unwrapped distance traveled by the ray, $G_{T}$ is the transmit antenna gain, $G_{R}$ is the received antenna gain, $P_{T}$ is the transmit power, $\lambda$ is the wavelength. We assume the ray undergoes $M$ transmissions and $K$ reflections before reaching the receiver; $t_{m}$ denotes the transmission coefficient for the $m^{\text {th }}$ transmission; and $r_{k}$ denotes the reflection coefficient for the $k^{t h}$ reflection. The relevant output of WISE for generating ELVIS inputs is $N$ received rays and their AOA (both azimuth and elevation angles), powers and delays.

\section{BACKWARD RAY TRACING IN ELVIS}

In ELVIS, only $N$ arrivals at the receiver which have the most received power are considered, to reduce complexity without sacrificing performance. Each of these $N$ rays is traced backward to the wall with which it last interacted. In an interaction, the ray could have been reflected from or transmitted through the wall. The point of interaction is called the point of incidence. The reflected and transmitted rays are determined at the point of incidence. Assume a receiver at $A$ receives a ray from the point $B$ as shown in Figure 2 . To predict the path from which this ray comes, it launches a

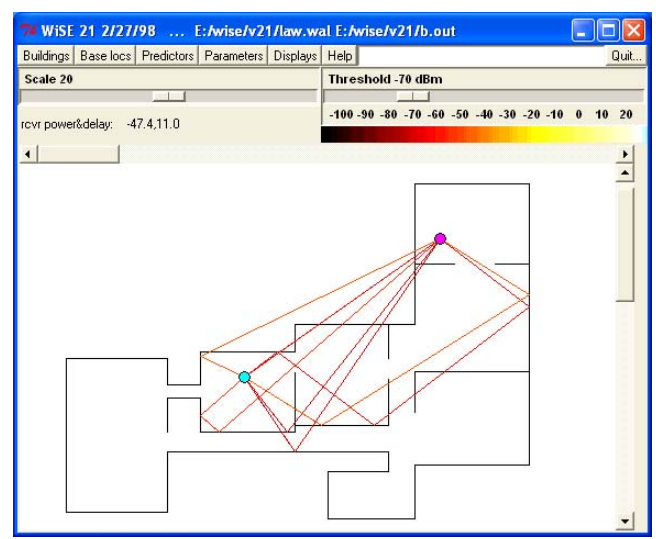

Fig. 1. WISE simulates radio paths between receiver and transmitter in a law office. Rays shown are projections onto a 2D plane

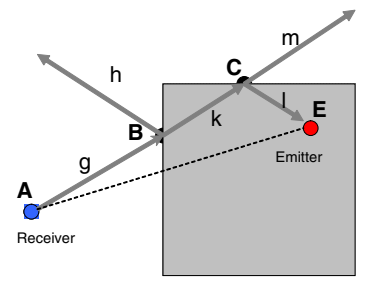

Fig. 2. Backward ray tracing: Each ray becomes a new arrival at the effective point of incidence

virtual ray $g$ towards $B$. This ray hits the wall at $B$, called the point of incidence. Now $B$ becomes an effective emitter from which the transmitted ray $k$ and reflected ray $h$ are emanated. The reflected ray $h$ doesn't hit any other wall, whereas the transmitted ray $k$ hits another wall at $C$, which becomes the effective source from which the reflected ray $l$ and transmitted ray $m$ are emanated. $m$ doesn't hit another wall, whereas $l$ goes through the emitter location $E$. Backward ray tracing is applied until stopping conditions are reached or the ray leaves the building. The same concept is applied for another arrival at $A$ to determine candidate intersection points, one of which could be a true emitter location. As we have seen in the previous example for backward ray tracing, we have to determine if the ray hits a wall and if so, where. Let's consider this problem in more detail for a $3 \mathrm{D}$ case. A rectangular wall in $3 \mathrm{D}$ is given in most general form by the equations:

$$
A x+B y+C z+D=0
$$

where $x \epsilon\left\{x_{\min }, x_{\max }\right\}, y \in\left\{y_{\min }, y_{\max }\right\}, z \epsilon\left\{z_{\min }, z_{\max }\right\}$ and $\mathbf{n}=(A, B, C)^{T}$ is the normal to the plane of the wall. The line on which a ray lies is given in parametric form as:

$$
(x, y, z)^{T}=\left(x_{0}, y_{0}, z_{0}\right)^{T}+t\left(x_{d}, y_{d}, z_{d}\right)^{T} .
$$

In our problem, we choose $\left(x_{0}, y_{0}, z_{0}\right)^{T}$ as the ray origin position vector, i.e, the point from which the ray emanates. $v=\left(x_{d}, y_{d}, z_{d}\right)^{T}$ is chosen as the unit vector in the direction of the ray and the length parameter $t(t>0)$ corresponds to the distance between the ray origin $\left(x_{0}, y_{0}, z_{0}\right)^{T}$ and any other 


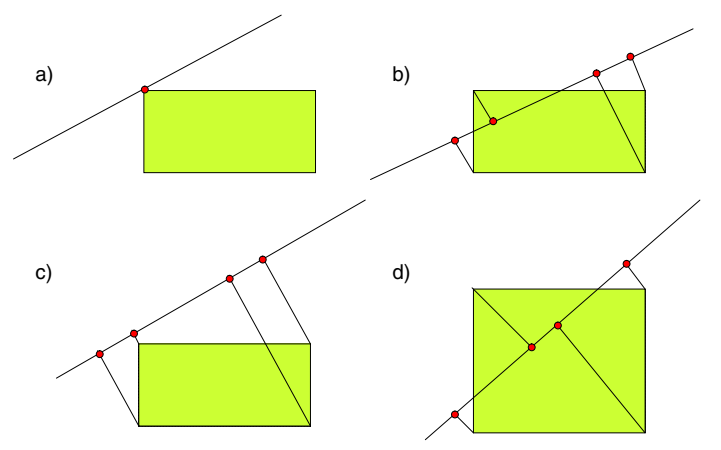

Fig. 3. Cases where, the ray and wall are on the same plane a) The ray intersects the wall only at a corner point. b) The ray intersects the wall. Only one of the most nearest points of the ray from the corners lie on the wall. c) The ray doesn't intersect the wall. None of the most nearest points of the ray from the corners lie on the wall. d) The ray intersects the wall. Two of the most nearest points of the ray from the corners lie on the wall.

point on the ray. To find the intersection point of ray and wall we substitute (3) into (2) obtaining:

$$
A x_{0}+B y_{0}+C z_{0}+t\left(A x_{d}+B y_{d}+C z_{d}\right)+D=0
$$

We solve for $t$ to determine the intersection point. Valid intersection point occurs if $t \geq 0$ and the constraints in (2) are satisfied. If they are, the value of $t$ may be used in (3) to find the point of incidence. We define two new variables:

$$
\begin{aligned}
& E=A x_{d}+B y_{d}+C z_{d} \\
& F=A x_{0}+B y_{0}+C z_{0} .
\end{aligned}
$$

Now we can write (4) as:

$$
F+t E+D=0
$$

$F$ is the scalar product of the normal of the plane with the position vector of the ray origin. $E$ is the scalar product of the normal of the plane with the direction vector of the line. If $F=0$, it means that position vector of ray origin $\left(x_{0}, y_{0}, z_{0}\right)^{T}$ is orthogonal to the normal of the plane. If $E=0$, direction vector of the line $\left(x_{d}, y_{d}, z_{d}\right)^{T}$ is orthogonal to the normal of the plane. We now have four cases:

- $E=0, F+D=0$ : Transmitter and ray are in the same plane as the wall. As seen in Figure 3, there are multiple possibilities which needs to be distinguished if the ray and wall lie in the same plane. We have to investigate further if the ray intersects the wall. For this we find the nearest point on the ray from each corner point. If at least one of those points lie on the wall we say the ray intersects the wall.

- $E=0, F+D \neq 0$ : Ray is parallel to plane but not on plane. It doesn't intersect the wall.

- $E \neq 0, F+D=0$ : Transmitter is on the plane but ray is not parallel to plane. If the coordinates of the transmitter fulfill the conditions in (2), the transmitter lies on the wall.

- $E \neq 0, F+D \neq 0$ : The line intersects the plane of the wall. The transmitter and the ray are not on the same plane as the wall.
For the case $E \neq 0, F+D \neq 0$ we get a nonzero t:

$$
t=-\frac{(D+F)}{E} .
$$

The ray intersects the plane if $t>0$. We can determine this intersection point from (3). The intersection point lies on the wall if the conditions of (2) are fulfilled.

\section{Emulating Degradations}

In reality, the ray differs from prediction due to unaccounted-for objects, such as furniture and people. Errors can also be caused by imprecision in the blueprint and wall materials. We treat the case of ideal conditions (no other objects, perfectly-known blueprint and materials), but we also treat the departures from ideal as discussed below. We assume, initially, that each receiver makes perfect measurements of time delays, arrival angles and ray amplitudes. To obtain realistic assessments, however, we then relax these assumptions by considering finite resolutions in delay and angles, and signal fading plus noise.

AOA imprecision arises because of the finite beamwidths, in azimuth and elevation, of the receive antennae. To simulate this effect, we quantize the AOA values using angular bins. The rays arriving within a bin are all assigned the same values of azimuth and elevation angle, e.g., for a 20-degree bin width all angular errors for rays lie within \pm 10 degrees.

TOA imprecision is caused by the finite bandwidth of the measured signal. We simulate TOA errors in a similar way, by using finite-width delay bins, with a common TOA assigned to all rays within the same bin. For both AOA and TOA, the quantizing errors can be widened to reflect the additional possible errors due to imprecise knowledge of building walls, materials and objects, as discussed above.

Quantization in azimuth angle, elevation angle and time delay can be seen as assigning each ray to one grid location on a $3 \mathrm{D}$ grid. We sum the ray powers within the same bin and assign the sum as the average ray power for that bin. At the end of this process, we choose the $\mathrm{N}$ bins with the largest ray power and we perform backward ray tracing.

We simulate the effect of fading and noise in the following way: For the $i^{t h}$ bin the received signal is given by

$$
y_{i}=s_{i}+n_{i},
$$

where $s_{i}$ is the complex signal sample and $n_{i}$ is a complex random noise sample. We assume $1 \mathrm{~W}$ transmit power, 4 $\mathrm{dB}$ noise figure, $300 \mathrm{~K}$ noise temperature and $300 \mathrm{MHz}$ bandwidth. Thus, $n_{i}$ is an i.i.d. with zero mean and a meansquare value of 3 picowatts.

Making the further assumption that signal and noise are uncorrelated, the received power in the $i^{\text {th }}$ grid is

$$
P_{R}=P_{s}+P_{n}
$$

where $P_{R}=E\left\{\left|y_{i}\right|^{2}\right\}, P_{s}=E\left\{\left|s_{i}\right|^{2}\right\}$ and $P_{n}=E\left\{\left|n_{i}\right|^{2}\right\}$. Degradations due to noise is simulated by generating a complex Gaussian noise sample, $n_{i}$, whose variance is $P_{n}$, and adding it to $s_{i}$ as in (9). 
We fade the signal amplitude by assuming a Rayleigh distribution. Each bin ray may in fact be a cluster of rays, due to both diffuse surfaces in the actual environment and the summing of multiple rays in the same bin. The assumed Rayleigh fading models the variability of the ray sum, though it may be on the pessimistic side. We will show results both with and without fading, so as to bracket reality. In the case of fading, $s_{i}$ is complex Gaussian with variance $P_{s}$ and $y_{i}$ is a complex Gaussian with variance $P_{s}+P_{n}$. Thus $P_{R}$ is an exponentially distributed random variable with mean $P_{s}+P_{n}$.

\section{ELVIS}

\section{A. Basic Algorithm}

ELVIS consists of three steps:

1) Backward ray tracing - For each arrival, we apply the backward ray tracing method separately. Power of any point along the back traced ray path is computed by inverting (1). Assume the ray has undergone $M$ transmissions and $K$ reflections through its path. We define a new variable $S$, which is the product of the $M$ transmission and $K$ reflection coefficients:

$$
S=\prod_{m=1}^{M} t_{m} \prod_{k=1}^{K} r_{k}
$$

The value of $S$ gets smaller with increasing $K$ and $M$, since $t_{m}, r_{k} \epsilon\{0,1\}$. The power $P$ at any point on the ray is predicted using

$$
P=\frac{4 \pi^{2}}{G_{T} G_{R} \lambda^{2}} \frac{l^{2}}{S} P_{R},
$$

$l$ is the total path the ray traveled between that point and the receiver. From (12) we see that $P$ increases with increasing $l, K$ and $M$. A ray received with less $P_{R}$ needs to be back propagated a greater distance and interact with more walls to reach the same expected power value $P_{T}$.

We save all backward-traced rays along with their powers and propagation delays. We stop backward ray tracing if any of the following occur: $P>P_{T} ; K$ or $M$ exceeds a prescribed limit; the ray leaves the building.

2) Determining candidate locations - Candidate locations are pairwise intersections of the back-traced rays. Under ideal conditions, emitter location is characterized by having multiple ray intersections with all backward ray traced powers equal to each other and to $P_{T}$ and with the same generation times.

3) Choosing the actual source location - As there are usually multiple candidate locations, there is a need to establish metrics for choosing the most likely one. The metrics could be based on additional measured parameters. Selection criteria can be based on weighted sums of the metrics. We consider the following metrics which are normalized between 0 and 1 :

a) Number of intersections in the vicinity: The regions with multiple candidate locations are more likely to contain the emitter location therefore the candidate locations in these areas have higher metric. Let $n_{i}$ denote the number of candidate locations which are maximum $x \mathrm{~m}$ away from the $i^{t h}$ candidate location. We define the first metric $m_{1, i}$ as:

$$
m_{1, i}=\frac{n_{i}}{\max _{k} n_{k}} .
$$

b) Predicted power difference between rays: At the true emitter location the predicted powers of intersecting rays are expected to be equal. Let $p_{i}$ denote the absolute power difference between intersecting rays at the $i^{t h}$ candidate location. We define $m_{2, i}$ as:

$$
m_{2, i}=1-\frac{p_{i}}{\max _{k} p_{k}} .
$$

c) Power difference between the expected transmit power $P_{T}$ and predicted power $P$ for each intersecting ray: At the emitter location the predicted and estimated power should be equal. Consider two rays intersecting at the $i^{\text {th }}$ candidate location. Let $\Delta p_{1, i}$ be first ray's power deviation from the expected power $P_{T}$ and $\Delta p_{2, i}$ second ray's power deviation from the expected power $P_{T}$ (Selecting the rays as first and second is arbitrary.) We define $m_{3, i}$ as:

$$
m_{3, i}=1-\frac{\Delta p_{i, 1}}{\max _{k} \Delta p_{k, 1}}
$$

and similarly comparing the second ray's power $P$ with $P_{T}$ :

$$
m_{4, i}=1-\frac{\Delta p_{i, 2}}{\max _{k} \Delta p_{k, 2}}
$$

d) Relative delay between arrivals: ELVIS does not depend on the arrival times of the rays. However, we assume the receiver is able to estimate delay differences among arriving rays. ELVIS can also estimate these arrival times for any given candidate location. Thus, the set of relative delays measured by the receiver can be compared against the set of relative delays computed for any candidate location. The difference comprises another possible metric. Assume two rays, $g$ and $h$, that arrive at the receiver at the receiver at times $t_{g}$ and $t_{h}$. By backward ray tracing we determine that they are intersecting at the $i^{t h}$ candidate location. Since we save by backward ray tracing for each ray the corresponding path from the receiver to that candidate location we can determine the unwrapped distance $d_{g}$ and $d_{h}$ from the receiver. We find the predicted propagation delays from $\Delta t_{g}=d_{g} / c$ and $\Delta t_{h}=$ $d_{h} / c$ where $c$ is speed of light. For $i^{\text {th }}$ candidate location we compute $z_{i}=\left|\left(t_{g}-t_{h}\right)-\left(\Delta t_{g}-\Delta t_{h}\right)\right|$. 
We define $m_{5, i}$ as:

$$
m_{5, i}=1-\frac{z_{i}}{\max _{k} z_{k}}
$$

ELVIS computes the weighted metric sum for each candidate location. The metrics can be weighted differently depending on the reliability of the measurement. The candidate location which has the highest metric sum is chosen. In this work, we weight the metrics equally.

\section{B. Multiple Receivers}

If the measurements are not perfect multiple receivers decrease the prediction error. We assume that the multiple receivers can communicate with a central processing unit which runs ELVIS. Alternatively, if we assume the emitter location doesn't change over the duration of sensing and subsequent processing by ELVIS, one could change the location of the same antenna setup and take multiple measurements instead of having multiple receivers.

Each receiver sends to the central processing unit the AOA of each incoming ray, TOA, received power and the coordinates of the receiver. The ELVIS algorithm for multiple receivers is the same as for the single receiver case, except for the ray selection. We select the $N$ strongest bin rays received among all antennas. To understand this, assume that there are a total of $K$ receivers and the $k^{t h}$ receiver reports $n_{k}$ rays. Out of the $\sum_{k=1}^{K} n_{k}$ rays, the top $N$ rays (in terms of received power) are selected. Thus, a receiver which is closer to the emitter is more likely to contribute with more rays, whereas the receivers far away contribute few, if any rays. Selecting the strongest rays in power among all received rays decrease the computational time significantly, since the number of the walls which a strong ray could reach by backward ray tracing is less than for a weaker ray. Increasing the number of receivers might decrease the prediction error, but it won't increase the computation time significantly since we choose a fixed number of rays independent of the number of receivers. Backward ray tracing rays from different receivers results in fewer intersections; thus, there are fewer candidate points, which further decreases the computation time.

\section{Simulation RESUlts}

For investigation of the overall performance in the whole Crawford Hill building, we fixed the receiver(s) and varied the location of the emitter. To obtain the sample points for emitter location, we have divided the building uniformly in 5 $\mathrm{m} \times 5 \mathrm{~m}$ grids whose centers give us 72 sample points. We have computed for each sample point the location error, i.e. the distance between predicted and true emitter location and determined the CDF (Cumulative Distribution Function). We choose $N=5$ in our simulations.

\section{A. Perfect Measurements}

First, we assume the receiver is able to resolve rays with exact measurements of angle, delay and power.

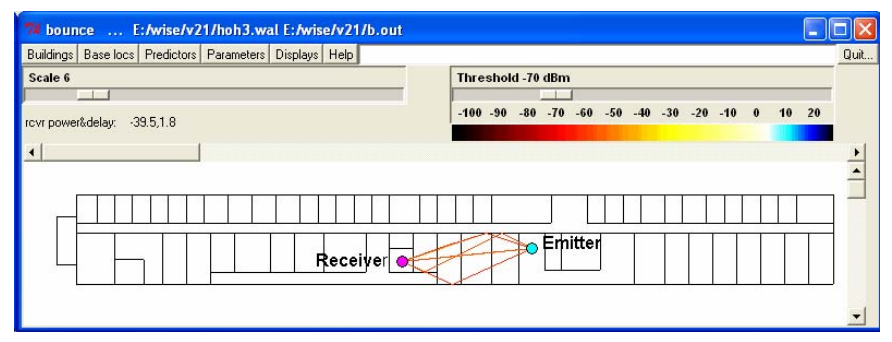

Fig. 4. WISE output: Radio paths between receiver and emitter in the Crawford Hill building. This building is about $120 \mathrm{~m}$ x15 m

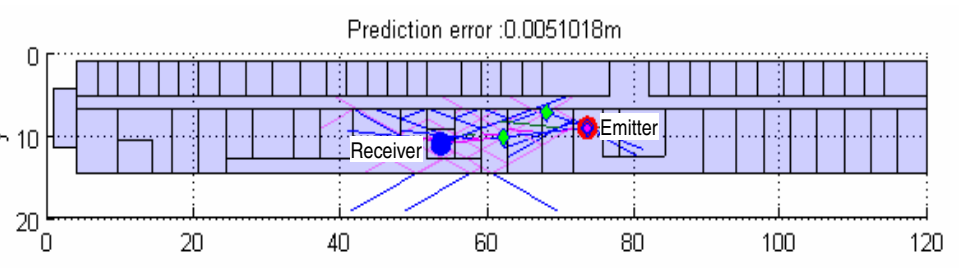

Fig. 5. ELVIS result: Prediction of emitter location using single receiver with perfect measurement of AOA, TOA and power

1) Single Receiver: Figure 4 shows the radio paths between emitter and receiver predicted by WISE. ELVIS predicts the location of this emitter within $5 \mathrm{~mm}$ as shown in Figure 5 . Figure 6 shows the CDF of the location error using single receiver. We see that, for $90 \%$ of the building, the location error is less than $2 \mathrm{~cm}$ with a maximum error of $9 \mathrm{~cm}$.

2) Multiple Receivers: Figure 6 shows the CDF of the location error using 6 receivers, which are placed at suitable positions both inside and outside building. We could localize over $90 \%$ of the building within $1 \mathrm{~cm}$ using multiple receivers, with a maximum error of $3.5 \mathrm{~cm}$. From these experiments, we conclude that, if the measurements are perfect, only one receiver is enough to do accurate localization, and that multiple receivers are effective in further reducing the location error.

\section{B. Degraded Measurements}

For angular degradation, we quantized the elevation and azimuth angles using 0, 10 and 20 degree quantization bins. For the temporal degradation, we quantized the TOA's of the rays using 0,10 , and $20 \mathrm{~ns}$ bins. We also distorted the signal amplitude to emulate the fading and add noise to the measurements. In the following CDF's $\mathrm{N}$ denotes degradation due to noise, $\mathrm{F}+\mathrm{N}$ denotes degradation due to noise and fading, $10 \mathrm{~ns}-20 \mathrm{~ns}$ denote the width of the quantization bin for degradation of TOA.

1) Single Receiver: In Figure 7, we compare the CDF of location error for various degradations by perfect measurement of the AOA. We see that the location error is more affected by fading than by degradation of the TOA. In the noise-only case we localize an emitter within $5 \mathrm{~m}$ over $93 \%$ of the building. Adding fading to the signal amplitude, we localize only $75 \%$ of the building within $5 \mathrm{~m}$ over. Using 10 degree angular bins we could localize only $30 \%$ of the building within $5 \mathrm{~m}$ for the best scenario. In Figure 8 we see that setting angular 


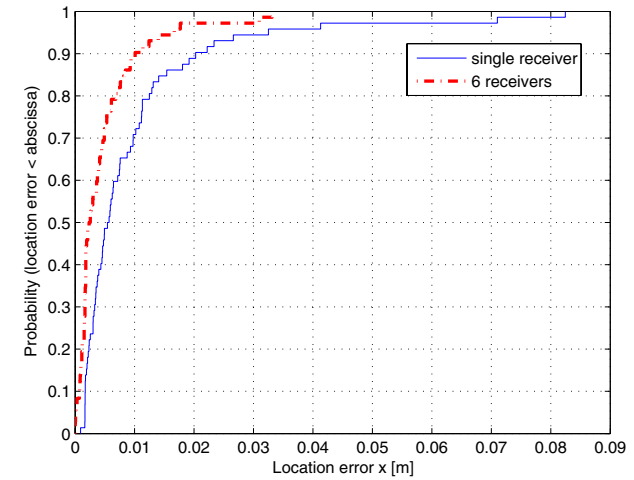

Fig. 6. CDF of Prediction Error by perfect measurement of power, TOA and AOA

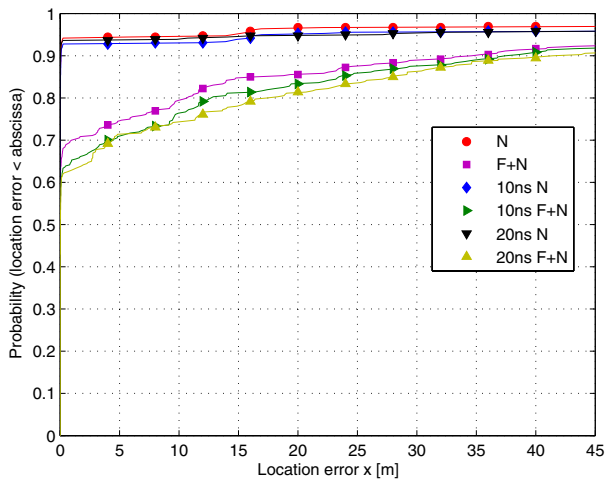

Fig. 7. CDF of Prediction Error by perfect measurement of AOA (single receiver) and various degradations

quantization bins to 20 degrees makes the performance of the localization algorithm even worse. Here, for the best scenario we can localize within $5 \mathrm{~m}$ over only $20 \%$ of the building. The most crucial component amongst AOA, TOA, fading and noise towards the prediction error of ELVIS is the angular error. The next most crucial component is fading, while TOA has the least effect on prediction error.

2) Multiple Receivers: Using 6 receivers and having perfect angular resolution the location errors are within $5 \mathrm{~m}$ over $90 \%$ of the building at worst. For angular bins of 10-degree width location errors are within $5 \mathrm{~m}$ over $60 \%$ of the building. For bins of 20-degree width, Figure 8 shows that location error within $5 \mathrm{~m}$ occur over only $48 \%$ of the building and location error within $10 \mathrm{~m}$ occur over $80 \%$. Also the spread over different cases $(\mathrm{N}, \mathrm{F}+\mathrm{N}$, etc.) is smaller with multiple receivers.

\section{CONCLUSION}

We have developed and simulated ELVIS, a backward ray tracing-based localization tool for indoor environments. We have determined that backward ray tracing can be effective even with degradations, especially if multiple receivers are used. We find that, if the angular resolution is high, ELVIS gives reliable results with a single receiver. At lower angular

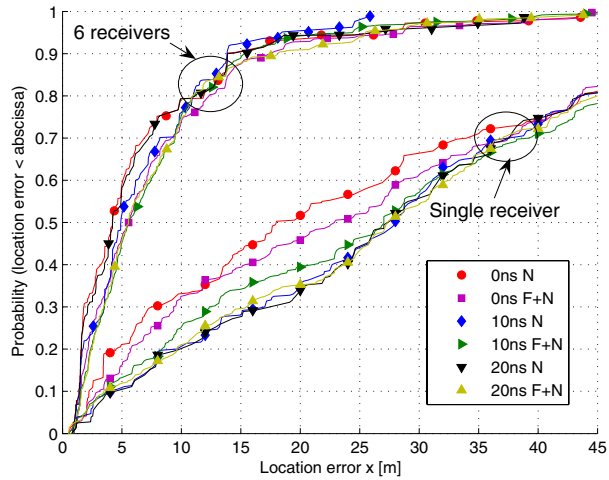

Fig. 8. CDF of Prediction Error using 20 degree angular bins and various degradations

resolution, multiple receivers are necessary to localize with good accuracy. Fading and noise are not major causes of degradation in most cases of interest.

We have simulated the measurements at the receiver using WISE. Further work could include (1) investigation of other metrics, to improve the performance; (2) investigation of the performance in other buildings; (3) adding other error sources, such as uncertainties in the blueprint and wall properties; and (4) verification of this technique through measurements.

While we find that the backward ray tracing technique in ELVIS produces highly accurate results using ideally accurate measurements, finite angular resolution is the primary source of degradation in accuracy. In the approach taken here the rays are traced back in the direction of the center of the angular bin on which they were received. Finite angular resolution can lead to rays being back-traced in wrong directions. With increased distance such rays may begin striking wrong walls and take paths that are not even in the same area as the transmitter. A possible remedy for this would be to back-trace multiple rays within each angular bin, allowing better candidate locations to be discovered.

\section{REFERENCES}

[1] A. L. E. L. V. Otsason, A. Varshavsky, "Accurate GSM indoor localization," UbiComp, pp. 141-158, 2005.

[2] S. Gezici, Z. Tian, G. Giannakis, H. Kobayashi, A. Molish, V. Poor, and Z. Sahinoglu, "Localization via Ultra-Wideband Radios," IEEE Signal Processing Magazine, vol. 22, no. 4, pp. 70-84, July 2005.

[3] P. Bahl and V. Padmanabhan, "Radar: An in-building RF-based user location and tracking system," IEEE Infocom, pp. 775-784, 2000.

[4] A. Madd, K. Bekris, A. Rudys, D. Wallach, and L. Kavraki, "On the feasibility of using wireless ethernet for indoor localization," Robotics and Automation, IEEE Transactions on, vol. 20, no. 3, pp. 555-559, June 2004.

[5] D. K. K. Sayrafian-Pour, "Indoor positioning using spatial power spectrum," PIMRC, 2005.

[6] P. Voltz and T. Lui, "A time domain backpropagating ray technique for source localization," The Journal of the Acoustical Society of America, vol. 95, no. 2, pp. 805-812, February 1994.

[7] S. Fortune, D. Gay, B. Kernighan, O. Landronand, R. Valenzuela, and M. Wright, "WISE design of indoor wireless systems: practical computation and optimization," IEEE Computational Science and Engineering, vol. 2, no. 1, pp. 58-68, 1995. 